\section{Non-narcotic analgesics associated with increased risk of hypertension}

Women who take high daily doses of acetaminophen and some nonsteroidal anti-inflammatory drugs (NSAIDs) have an elevated risk of developing incident hypertension, a prospective study carried out in the US has shown.

Forman and colleagues investigated a possible association between the dose of analgesic drugs used and incidence of hypertension among 1,903 women aged 51-77 years who participated in the Nurses' Health Study I and 3,200 women aged 34-53 years from the Nurses' Health Study II. Women included in the study had no history of hypertension at baseline. They completed detailed questionnaires about their use of acetaminophen, aspirin or other NSAIDs, including average daily doses and indications for their use. Information was also gathered about relevant confounders such as alcohol intake and history of smoking. Study participants were then followed up prospectively to find out whether they developed hypertension.

Women in both age-groups who took at least $500 \mathrm{mg}$ of acetaminophen daily were almost twice as likely to develop hypertension as those who did not use acetaminophen. Among women aged $51-77$ years who used at least $400 \mathrm{mg}$ NSAIDs daily, the risk of hypertension increased by almost $80 \%$, whereas for younger women (aged 34-53 years) the risk of hypertension was $60 \%$ higher compared with individuals who did not use NSAIDs. Interestingly, high doses of aspirin were not associated with an increased risk of developing hypertension in either age-group.

According to the researchers, these results have significant public health implications and suggest that acetaminophen and NSAIDs should be used with greater caution.

Claire Braybrook

Original article Forman JP et al. (2005) Non-narcotic analgesic dose and risk of incident hypertension in US women. Hypertension 46: 500-507

\section{Low-dose rosuvastatin for hypercholesterolemic patients with the metabolic syndrome}

While statin therapy is an obvious part of combined drug treatment for the optimum management of combined hyperlipidemia, the efficacy of statin therapy, and a comparison of the effects of different statins, have not been investigated in patients with the metabolic syndrome.

This post hoc analysis of the randomized MERCURY I trial compared the effects of four statin drugs on the lipid profiles of 3,140 individuals with and without the metabolic syndrome. Only patients with coronary or other atherosclerotic disease, or type 2 diabetes, were eligible for the study, and were assigned one of five daily treatment regimens: $10 \mathrm{mg}$ rosuvastatin, $10 \mathrm{mg}$ or $20 \mathrm{mg}$ atorvastatin, $20 \mathrm{mg}$ simvastatin or $40 \mathrm{mg}$ pravastatin.

LDL cholesterol, total cholesterol and nonHDL cholesterol levels were significantly lower in patients who received $10 \mathrm{mg}$ rosuvastatin than in patients treated with other statin therapies, except $20 \mathrm{mg}$ atorvastatin. Triglyceride levels were also significantly reduced and HDL cholesterol levels raised in the $10 \mathrm{mg}$ rosuvastatin group, regardless of the presence of the metabolic syndrome. Importantly, more patients reached the ideal LDL cholesterol level with low-dose rosuvastatin than other treatment regimens.

Stender et al. conclude that statins improve the lipid profile of high-risk hypercholesterolemic patients with and without the metabolic syndrome and that low-dose rosuvastatin was the most effective treatment regimen.

Hannah Camm

Original article Stender S et al. (2005) Comparison of rosuvastatin with atorvastatin, simvastatin and pravastatin in achieving cholesterol goals and improving plasma lipids in hypercholesterolaemic patients with or without the metabolic syndrome in the MERCURY I trial. Diabetes Obes Metab 7: 430-438

\section{Risks and benefits of warfarin plus aspirin therapy for patients with acute coronary syndromes}

A recent meta-analysis indicates that warfarin plus aspirin therapy has a beneficial effect on cardiovascular outcome for some patients with acute coronary syndromes, despite an increased risk of bleeding.

Although there is some evidence that the addition of warfarin to standard aspirin therapy can reduce the incidence of 